\title{
METHODS \& DESIGNS \\ Monitoring visual activity in infant rhesus monkeys: Method and calibration
}

\author{
MORTON J. MENDELSON \\ McGill University, Montreal, Quebec H3A 1BI, Canada \\ MARSHALL M. HAITH \\ University of Denver, Denver, Colorado 80210 \\ and \\ PATRICIA S. GOLDMAN-RAKIC \\ Yale University, New Haven, Connecticut 06520
}

\begin{abstract}
This paper describes the use of an infrared corneal reflection technique to monitor visual activity in infant rhesus monkeys. To establish the validity of the method, calibration data were collected and a mathematical correction for systematic sources of error was used.
\end{abstract}

Behavioral research on vision in infant monkeys has typically been limited to paradigms using motor responses and learning techniques (see Boothe \& Sackett, 1975, for a review). These methods, however, are not useful for studying very young infants, with the notable exception of the forced-choice preferential looking paradigm (e.g., Teller, Regal, Videen, \& Pulos, 1978). Although preference studies can provide valuable information, they demand that questions be answerable in terms of a discrimination. Methods of monitoring eye movements and fixations are needed to investigate visual activity in detail or to answer questions about strategies of visual search.

Investigators have previously studied eye movements and fixations in adult monkeys (e.g., Cowey, 1963), and in human infants (e.g., Haith, 1980; Mendelson \& Haith, 1976; Salapatek, 1975). Here, we outline a method for recording eye movements in infant monkeys using infrared corneal reflection videotape recording (Haith, 1969).

It has been widely observed that the uncorrected

The authors are extremely grateful to the following individuals. Mary Ann Goodwyn patiently scored the data. Philippe Grall wrote the fixation correction program. Jean-René Duhamel and Zeena Yaffe assisted with the computer work. Fran Aboud, Irv Binik, and Beverley Katz Mendelson commented on an earlier draft. Finally, Giovanna LoCascio diligently typed and retyped the manuscript. The data collection, which was conducted in P. S. Goldman-Rakic's laboratory at NMH, Bethesda, Maryland, was supported by ADAMHA-NIMH intramural funds and by an NSERC (Canada) postdoctoral fellowship to M. J. Mendelson. The data analysis was funded by Grant MH23412 (M. M. Haith, principal investigator) and by Grant A-6347 from NSERC (Canada) (M. J. Mendelson, principal investigator). Reprint requests should be addressed to Morton J. Mendelson at the Psychology Department, McGill University, 1205 Docteur Penfield Avenue, Montreal, Quebec H3A 1B1, Canada. corneal reflection technique provides only an approximation of the direction of visual regard (Bullinger, 1974; Bullinger \& Kaufman, 1977; Maurer, 1975; Salapatek, 1975; Slater \& Findlay, 1972, 1975). There are two principal sources of error in the technique: First, the visual and optical axes of the eye do not coincide perfectly; that is, a line drawn through the center of the pupil and lens does not strike the retina at the fovea. Second, the curved plane of the cornea (which produces the corneal reflection) does not coincide with the plane of the pupil. The positional difference of these planes produces parallax, and the parallax varies as a function of the optical axis of the eye relative to the axis of the camera. Systematic error is therefore introduced into the scoring procedure. When fixations are in the temporal visual field, fixations are scored more to the temporal side than is actually the case. As fixations move toward and into the nasal side, the error decreases, disappears, and then reverses so that fixations are scored more nasal than is actually the case.

Bullinger and Kaufman (1977) derived two complicated formulas to deal with these sources of error as well as with errors due to idiosyncrasies in individual subject's eyes. The functions are

$$
\begin{aligned}
& X^{\prime}=f(X, A, R, P, \sin \alpha, D) \\
& Y^{\prime}=f\left(Y, X, X^{\prime}, \sin \alpha\right),
\end{aligned}
$$

where $\mathrm{X}^{\prime}$ and $\mathrm{Y}^{\prime}$ are the scored (i.e., uncorrected) coordinates of fixation, $X$ and $Y$ are the corrected coordinates, $A$ is the axial length of the eye, $R$ is the radius of curvature of the cornea, $P$ is the distance between the apex of the cornea and the plane of the pupil, $\alpha$ is the angle between the optical and visual axes, 
and $\mathrm{D}$ is the distance between the center of rotation of the eye and the plane of the stimulus. Unfortunately, the formulas are too complicated to be solved readily for corrected coordinates as a function of scored coordinates. Nevertheless, Bullinger and Kaufman proposed a procedure for correcting scored fixations that involves constructing a "look-up" table relating scored and corrected coordinates. Table construction is based on information about $\mathrm{X}^{\prime}, \mathrm{Y}^{\prime}, \mathrm{X}, \mathrm{Y}, \mathrm{R}$, and $\alpha$ that is obtained from calibration data and on estimates of $P$ and A. Once constructed, the table can be used to corrèct scored data from scanning studies. We describe here the use of this error-correction technique with calibration data obtained from infant monkeys to establish the validity of the procedure for future work.

\section{METHOD}

\section{Subjects}

Fifteen infant rhesus monkeys (Macaca mulatta) provided calibration data. Each monkey was observed once a week for at least the first 7 weeks of life. Here, we will consider data obtained from Weeks 1,3 , and 7 . When it was impossible to get reasonable calibration data on a particular week (for four monkeys in Week 1 and for one monkey in Week 3), data from an adjacent week were used.

\section{Apparatus}

Monitoring visual acuity. The method for monitoring visual activity, which involved infrared corneal reflection videotape recording, was adapted from work with human infants (Haith, 1969). A diagram of the recording equipment is shown in Figure 1. An infant monkey was held by the experimenter so it faced a stimulus panel $23.5 \mathrm{~cm}$ away. The panel was illuminated by two incandescent tubes (not in Figure 1) mounted on either side above the monkey's head. A video camera mounted horizontally behind the stimulus panel recorded the monkey's eye. The camera was Shibaden Model HV16A, equipped with a 75-mm f: 1.4 lens, a +3-diopter close-up lens, and an Amperex 5-1202 infrared silicon tube. Reference lights (Bausch \& Lomb Nicholas microscope substage illuminators 31-32-53) were also mounted behind the panel, three on either side of the camera. The light beams passed through Polaroid HN7 and Corning CS7 69 filters that eliminated all visible, and longer, hot wavelengths. $A$ band of wavelengths $800-1,100 \mathrm{~nm}$ was transmitted. The resulting infrared beams converged at the monkey's eye and the reflection of the infrared sources on the monkey's comea provided pre-

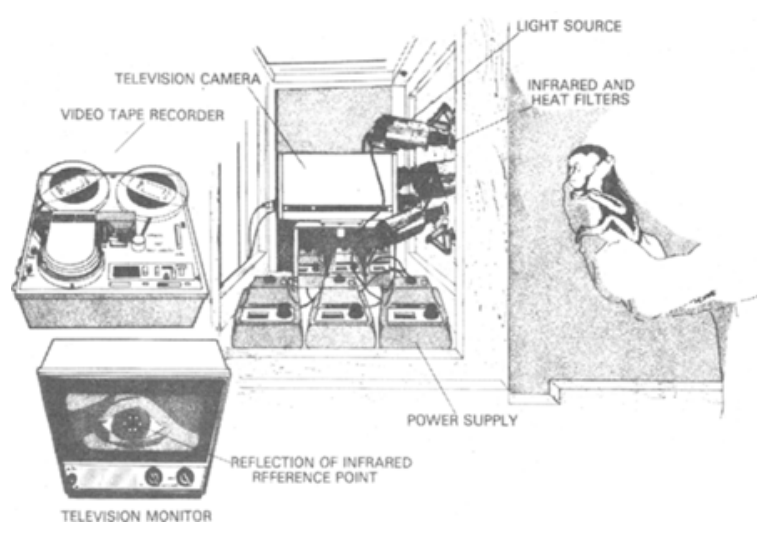

Figure 1. Apparatus for monitoring visual activity in infant monkeys.

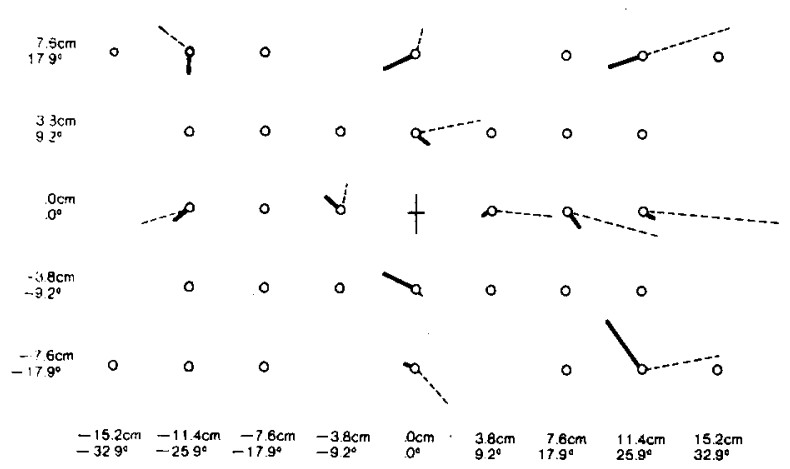

Figure 2. Schematic representation of the calibration lights (open circles drawn to scale). The cross represents the middle of the visual field. The marginals are the deviations from the center of each row and column of lights in centimeters, as well as in degrees of visual angle for the $23.5 \mathrm{~cm}$ viewing distance. The ends of the dashed lines are scored fixations for one monkey at Week 7; the ends of the thick lines are the corrected fixations.

calibrated reference markers to be used in scoring fixation positions. The center of the camera lens was considered the origin of a Cartesian coordinate system. The centers of the illuminator filaments were imaged at the plane of the stimulus panel at locations corresponding to the following $X-Y$ positions (in centimeters): $+17.2, .0 ;+5.1,+6.4 ;+5.1,-6.4 ;-17.2, .0$; $-5.1,-6.4 ;-5.1,+6.4$. A Sony CVM 112 monitor provided an image of the monkey's eye, and a video record was obtained with a Sony AV3600 tape recordex.

\section{Stimuli}

The calibration panel consisted of a black Plexiglas board with holes for the infrared marker lights and the television camera. There were 34 green calibration lights placed in an incomplete matrix over the board (shown schematically in Figure 2). The lights were $5 \mathrm{~cm}$ wide and were placed, when possible, every $3.8 \mathrm{~cm}$. In Figure 2, the Cartesian coordinates of the rows and columns are indicated both in centimeters from center and in degrees of visual angle for the $23.5-\mathrm{cm}$ viewing distance used. The lights could be individually flashed or illuminated by manually operating a button box.

\section{Procedure}

We tried to obtain a record of each monkey fixating as many known points as possible, which proved to be quite difficult, since infant monkeys did not readily direct their gaze to flashing or illuminated lights. For each calibration point, the monkey was held in the apparatus and a light was intermittently flashed or illuminated until the animal was judged to have fixated. Fixations involved a steady and relatively prolonged look at the light and were often accompanied by marked constriction of the pupil. If the monkey looked at the calibration light, the animal was removed from the apparatus, and the tape was reviewed to verify that a fixation had occurred. If the monkey did not fixate within approximately $20 \mathrm{sec}$, it was removed from the apparatus, the tape was rewound, and the procedure was repeated for a different light. In general, it was possible to obtain a record of 6 to 12 fixations in one or two $1 \mathrm{~h}$ sessions.

\section{Scoring}

A research assistant experienced with eye movement records scored the data. For each segment of tape, she judged whether the monkey had fixated the calibration point immediately prior to its removal from the apparatus. Only those fixations judged to be on target were scored. Thus, data were used only when the experimenter and the assistant agreed on the fixation.

A diagram of the apparatus for measuring fixation position is shown in Figure 3. Single "frames" of the video record were sampled manually when the monkey fixated the light. Each 


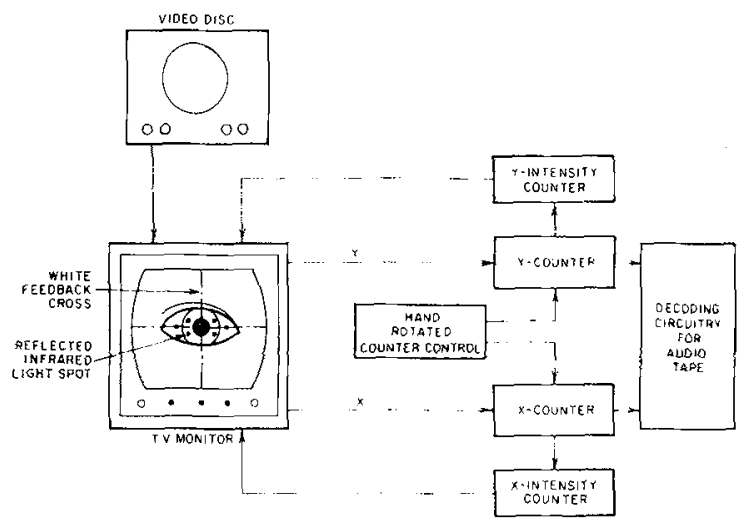

Figure 3. Schematic of apparatus for scoring fixation position.

frame was, in turn, displayed on an 18 -in. $(45.7-\mathrm{cm})$ Conrac video monitor. Also displayed on the screen were a horizontal and a vertical cross hair whose positions were controlled by manual potentiometers (Haith, 1969). The positions of the cross hairs were held in electronic counters and recorded on audio tape (Lentz \& Haith, 1969).

For a given frame, the scorer first defined a rectangie around the pupil that ultimately provided a measure of pupil center. The scorer set the cross hairs at the top and left margins of the pupil and then set them at the bottom and right margins. Finally, the scorer set the cross hairs at the infrared reflection nearest the center of the pupil. For each setting, a button was pressed that recorded the $X-Y$ positions of the cross hairs. The data on audio tape were analyzed by computer, yielding uncorrected coordinates of fixation for each frame based on a Cartesian system with the center of the stimulus panel as the origin.

\section{Estimating the Parameters for the Correction Equations}

Uncorrected coordinates. As many frames as possible of each fixation were sampled from the continuous video record. These were scored, and the mean $X$ and $Y$ locations were used as the uncorrected coordinates $\left(\mathrm{X}^{\prime}\right.$ and $\left.\mathrm{Y}^{\prime}\right)$ for the calibration point.

Axial length. The axial length for five monkeys (M. mulatta and $M$. arctoides) within the age range of this study were provided by Wiesel (Note 1). Ultrasound measurements indicated that the monkeys had a mean axial length of $14 \mathrm{~mm}$ (range: $14-15 \mathrm{~mm}$ ). The equations were tested using various axial lengths around the value of $14 \mathrm{~mm}$, and fairly wide fluctuations in the parameter had little effect on the computation. The $14-\mathrm{mm}$ value was therefore adopted for all monkeys at all ages.

Radius of curvature of the cornea. Ball bearings of various diameters were videotaped in the apparatus at the location of the animal's eye to find a radius of curvature that corresponded to the cornea. The video image of the bearings contained the reflections of the infrared sources. Frames of the record were sampled and displayed on the scoring moritor, and the distances between the infrared reflections were measured. For each monkey at each age, the distances between the infrared reflections on the cornea were also obtained. These were related to the measurements for various ball bearings, and the radius of curvature of the cornea was calculated by interpolation. The mean values were $5.5 \mathrm{~mm}, 5.7 \mathrm{~mm}, 5.9 \mathrm{~mm}$ at Weeks 1,3 , and 7. Again, a test of the effect of fairly wide fluctuations around the actual value obtained for a monkey revealed little effect on the computations. In the correction equation, the radius of curvature is related to the axial length, and since we used a single value for axial length across the three ages, we also used the mean radius of curvature for each monkey computed across the three ages.

Angle between the optical and visual axes. When the camera is located in the central axis of the visual field and the animal fixates straight ahead, the scoring error reflects the discrepancy between the optical and visual axes (i.e., Angle $\alpha$ ). Therefore, estimates of $\alpha$ for the horizontal axis can be obtained directly from calibrations for points directly on the vertical axis (i.e. $X=0)$. The mean $X$ value obtained for calibration points straight ahead was computed for each monkey at each age, and it was used to calculate Angle $\alpha$. The mean values of $\alpha$ were $6.9,6.7$, and 6.3 deg at Weeks 1,3 , and 7 .

We were concerned about the accuracy of our estimates of $\alpha$. Monkeys often had only one or two calibration points straight ahead, and errors in the scored position of these limited number of points would substantially alter the corrections. Therefore, the estimate of $\alpha$ for each monkey was adjusted at each age by averaging the estimated value and the mean value for the particular age. For example, a monkey whose value of $\alpha$ was estimated at $8.1 \mathrm{deg}$ during Week 1 was given a value of $(8.1+6.9) / 2=7.5 \mathrm{deg}$. Monkeys providing no calibration points straight ahead were given the group mean for $\alpha$ at the appropriate age.

The distance between the apex of the comea and the plane of the pupil (P). An equation for $\mathbf{P}\left(\mathrm{D}_{2}\right.$ in Bullinger \& Kaufman, 1977) was determined from the correction equation and was applied to each calibration point. Thus, each monkey provided several estimates of $\mathrm{P}$ at each age; the mean across all estimates was used as the appropriate value. The mean values at each of the three ages was $2.5 \mathrm{~mm}$. In cases in which the estimates varied considerably from calibration point to calibration point or in which there were no calibration data, the estimate of $P$ obtained at an adjacent age was used.

\section{RESULTS AND DISCUSSION}

Figure 2 shows calibration data obtained from a monkey during Week 7 . The monkey fixated 12 of the lights, and the scored fixation points are indicated at the end of the dashed lines. The mean deviation of the scored fixations from the lights was $6.4 \mathrm{deg}$. The correction procedure was applied to these data, and the adjusted fixation points are indicated at the end of the thick lines. The mean deviation of the adjusted fixations from the lights was $2.9 \mathrm{deg}$.

Figure 4 presents a summary of calibration data for the group of 15 monkeys. Each panel presents a histogram of calibration points deviating from the actual positions of the lights by amounts indicated on the abscissa. The data are graphed in bins whose upper bounds are indicated (e.g., 2 deg means $0-2 \mathrm{deg}, 4 \mathrm{deg}$ means $2.14 \mathrm{deg}$, etc.). Data from Weeks 1, 3, and 7 are graphed, respectively, in the top two, middle two, and bottom two panels, although data from an adjacent week were substituted or added in some cases. The panels on the left show the data for scored fixations (i.e., before correction) and the panels on the right show the data for the corrected fixations. The number of calibration points and the mean deviation for each of the six data sets are indicated in the upper right-hand corner of each panel.

At every age, the mean deviation after correction (4.2, 4.3, and $3.5 \mathrm{deg}$ for Weeks 1,3 , and 7) was considerably lower than the mean deviation prior to correction $(6.4,7.0$, and $5.4 \mathrm{deg})$. At every age, more than $75 \%$ of the corrected fixations were within 6 deg of the calibration light. Some of the errors even after correction were quite large (i.e., greater than $10 \mathrm{deg}$ ). These probably reflected inaccurate judgments of when the 


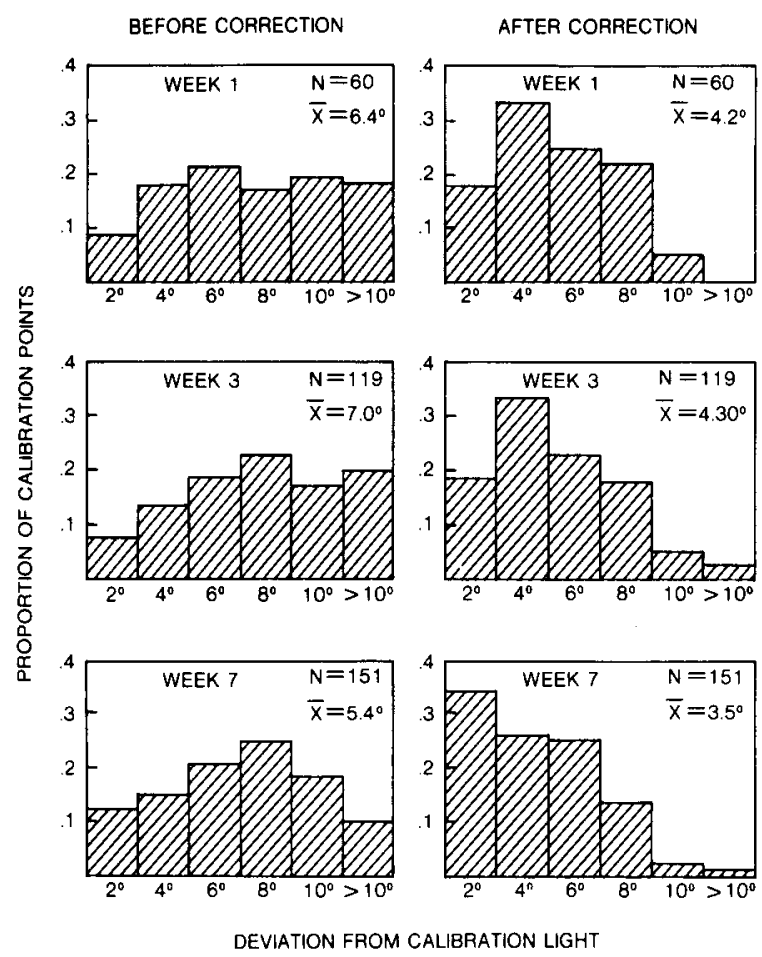

Figure 4. Histograms of the deviations from the expected fixation points for uncorrected and corrected data at each age.

monkeys fixated, rather than problems with the correction procedure. This observation is supported by the decreased variability in the errors with age. With older monkeys, it was easier to judge when the monkeys fixated the calibration light.

Further advances in accuracy might be possible using more sophisticated eye-tracking techniques. Even without improvements, the method can provide valuable information about vision in infant monkeys. Future reports will describe studies in which we monitored eye movements in the dark and while rhesus infants looked at geometric forms (Mendelson, Haith, \& Goldman, Note 2) and social stimuli (Mendelson, Haith, \& Goldman-Rakic, in press). In addition, we have applied these procedures to questions about the effects of early CNS lesions on scanning. The described technique provides further opportunities for studying the role of visual activity in perceptual and social development, as well as how physiological or traumatic manipulations affect vision in infant primates.

\section{REFERENCE NOTES}

1. Wiesel, T. N. Personal communication, May 23, 1978.

2. Mendelson, M. J., Haith, M. M., \& Goldman, P. S. Scanning of compound geometric forms in infant rhesus monkeys. Paper presented at the International Conference on Infant Studies, New Haven, Connecticut, March 1980.

\section{REFERENCES}

Boothe, R., \& Sacketr, G. Perception and learning in infant rhesus monkeys. In G. H. Bourne (Ed.), The rhesus monkey (Vol. 1). Anatomy and physiology. New York: Academic Press, 1975.

Bullinaer, A. L'enregistrement des mouvements oculaires par le reflet cornéen. Analyses des erreurs systematiques de la localisation du regard. Perception, 1974, 3, 355-360.

Bullinger, A., \& Kaufman, J.-L. Technique d'enregistrement et d'analyse des mouvements oculaires. Perception, 1977, 6, 345-353.

Cowey, A. The basis of a method of perimetry with monkeys. Quarterly Journal of Experimental Psychology, 1963, 15, 81-90.

HAITH, M. M. Infrared television recording and measurement of ocular behavior in the human infant. American Psychology, 1969, 24, 279-282.

HAiтH, M. M. Rules that babies look by: The organization of newborn visual activity. Hillsdale, N.J: Erlbaum, 1980.

LENTZ, R., \& HAITH, M. M. Audiotape storage of experimental data: An application to tachistoscopic research with children. Behavior Research Methods \& Instrumentation, 1969, 1, 273-275.

Maunz r, D. Infant visual perception: Methods of study. In L. B. Cohen \& P. Salapatek (Eds.), Infant perception: From sensation in cognition (Vol. 1). Basic visual processes. New York: Academic Press, 1975.

Mendelson, M. J., \& Haith, M. M. The relation between audition and vision in the human newborn. Monographs of the Society for Research in Child Development, 1976, 41(4, Serial No. 167).

Mendelson, M. J., Haith, M. M., \& Goldman-Rakic, P. S. Scanning of faces and responsiveness to social cues in infant rhesus monkeys. Developmental Psychology, in press.

Sal.apatex, P. Pattern perception in early infancy. In L. B. Cohen \& P. Salapatek (Eds.), Infant perception from sensation to cognition (Vol. 1): Basic visual processes. New York: Academic Press, 1975.

Slater, A. M., \& Findlay, J. M. The measurement of fixation positions in the newborn baby. Journal of Experimental Child Psychology, 1972, 14, 349-364.

Slater, A. M., \& Findlay, J. M. The corneal reflection technique and visual preference method: Sources of error. Journal of Experimental Child Psychology, 1975, 20, 240-247.

Teller, D. Y., Regal, D. M., Videen, T. O., \& Pulos, E. Development of visual acuity in infant monkeys (Macaca nemestrina) during the early postnatal weeks. Vision Research, 1978, 18, 561-566.

(Received for publication June 19, 1981; revision accepted July 28,1981 .) 